\title{
Association of Drug-Metabolizing Enzyme and Transporter Gene Polymorphisms and Lipid-Lowering Response to Statins in Thai Patients with Dyslipidemia
}

\author{
Natchaya Vanwong $\mathbb{D}^{1,2}$, Sayanit Tipnoppanon', Chalitpon Na Nakorn ${ }^{3}$, Pornpen Srisawasdi (D) ${ }^{4}$, \\ Punyanuch Rodcharoen ${ }^{4}$, Sadeep Medhasi ${ }^{5}$, Pajaree Chariyavilaskul (iD) ${ }^{2,6,7}$, \\ Sarawut Siwamogsatham ${ }^{2,8,9}$, Yongkasem Vorasettakarnkij ${ }^{2,9}$, Chonlaphat Sukasem ${ }^{10-12}$

\begin{abstract}
'Department of Clinical Chemistry, Faculty of Allied Health Sciences, Chulalongkorn University, Bangkok, Thailand; ${ }^{2}$ Cardiovascular Precision Medicine Research Group, Special Task Force of Activating Research (STAR), Chulalongkorn University, Bangkok, Thailand; ${ }^{3}$ Department of Clinical Pharmacy, Faculty of Pharmaceutical Sciences, Prince of Songkla University, Songkhla, Thailand; ${ }^{4}$ Division of Clinical Chemistry, Department of Pathology, Faculty of Medicine, Ramathibodi Hospital, Mahidol University, Bangkok, Thailand; ${ }^{5}$ Department of Transfusion Medicine and Clinical Microbiology, Faculty of Allied Health Sciences, Chulalongkorn University, Bangkok, Thailand; ' ${ }^{6}$ Department of Pharmacology, Faculty of Medicine, Chulalongkorn University, Bangkok, Thailand; ${ }^{7}$ Clinical Pharmacokinetics and Pharmacogenomics Research Unit, Chulalongkorn University, Bangkok, Thailand; ${ }^{8}$ Chula Clinical Research Center, Faculty of Medicine, Chulalongkorn University, Bangkok, Thailand; ${ }^{9}$ Department of Medicine, Faculty of Medicine, Chulalongkorn University, Bangkok, Thailand; ${ }^{10}$ Division of Pharmacogenomics and Personalized Medicine, Department of Pathology, Faculty of Medicine Ramathibodi Hospital, Mahidol University, Bangkok, Thailand; "'Laboratory for Pharmacogenomics, Somdech Phra Debaratana Medical Center (SDMC), Ramathibodi Hospital, Bangkok, Thailand; ${ }^{2}$ Pharmacogenomics and Precision Medicine, The Preventive Genomics \& Family Check-up Services Center, Bumrungrad International Hospital, Bangkok, Thailand
\end{abstract}

Correspondence: Chonlaphat Sukasem, Division of Pharmacogenetics and Personalized Medicine, Department of Pathology, Faculty of Medicine, Ramathibodi Hospital, Mahidol University, Bangkok, 10400, Thailand, Tel +66-2-200-433I, Fax +66-2-200-4332, Email chonlaphat.suk@mahidol.ac.th

Purpose: Statins are increasingly widely used in the primary and secondary prevention of cardiovascular disease. However, there is an inter-individual variation in statin response among patients. The study aims to determine the association between genetic variations in drug-metabolizing enzyme and transporter (DMET) genes and lipid-lowering response to a statin in Thai patients with hyperlipidemia.

Patients and Methods: Seventy-nine patients who received statin at steady-state concentrations were recruited. Serum lipid profile was measured at baseline and repeated after 4-month on a statin regimen. The genotype profile of 1936 DMET markers was obtained using Affymetrix DMET Plus genotyping microarrays.

Results: In this DMET microarray platform, five variants; SLCO1B3 (rs4149117, rs7311358, and rs2053098), QPRT (rs13331798), and SLC10A2 (rs188096) showed a suggestive association with LDL-cholesterol-lowering response. HDL-cholesterol-lowering responses were found to be related to CYP7A1 gene variant (rs12542233). Seven variants, SLCO1B3 (rs4149117, rs7311358, and rs2053098); SULT1E1 (rs3736599 and rs3822172); and $A B C B 11$ (rs4148768 and rs3770603), were associated with the total cholesterol-lowering response. One variant of the $A B C B 4$ gene (rs2109505) was significantly associated with triglyceride-lowering response.

Conclusion: This pharmacogenomic study identifies new genetic variants of DMET genes that are associated with the lipid-lowering response to statins. Genetic polymorphisms in DMET genes may impact the pharmacokinetics and lipid-lowering response to statin. The validation studies confirmations are needed in future pharmacogenomic studies.

Keywords: drug-metabolizing enzymes, drug transporters, gene polymorphisms, statin

\section{Introduction}

Dyslipidemia is the main risk factor for first and recurrent atherosclerotic cardiovascular disease (ASCVD) events. Atherosclerotic cardiovascular disease includes coronary artery disease, cerebrovascular disease, and peripheral artery

Received: 5 November 2021 Accepted: 4 February 2022

Published: 17 February 2022 
disease, all of which can lead to death if left untreated. It is one of the major causes of death globally and the top cause of death in Thailand. ${ }^{1}$ Statins (HMG-CoA reductase inhibitors) are drugs that are effective for reducing lipid levels and ASCVD prevention. ${ }^{2,3}$ However, there is an inter-individual variation in statins' response among patients. It has been reported that $48.2 \%$ of the patients did not reach the therapeutic low-density lipoprotein (LDL) cholesterol target, and only $35.5 \%$ of the patients achieved the therapeutic goal. ${ }^{4,5}$ The variation of individual responses to statin treatment may not only be owing to environmental factors such as medication adherence, exercise, and diet but also because of genetic factors. Polymorphisms of genes that involve in the regulation of lipid metabolisms such as apolipoprotein E (apo E), cholesteryl ester transfer protein (CETP), and liver X receptors (LXR) have been reported to correlate with the efficacy of statin and susceptibility to cardiovascular disease. ${ }^{6-11}$ Several studies have revealed that the genetic variants of the pharmacokinetics-related genes result in the diversity of statin efficacy among individuals. ${ }^{12-14}$ Since statins have the primary site of action and metabolism in the liver, where they inhibit HMG-CoA reductase, the genetic variations of Phase I and II drug-metabolizing enzymes and drug transporter genes may have a critical role in the concentration of statins within hepatocytes and plasma, and consequently clinical efficacy to the treatment of statin. Genetic variations on influx transporters and efflux transporters such as solute carrier organic anion transporter family, member $1 B 1$ (SLCO1B1), ATP binding cassette subfamily B member 1 (ABCB1), and ATP binding cassette subfamily $G$ member 2 (ABCG2) genes polymorphisms have been reported to be associated with the alteration of the pharmacokinetics of statin transport and the reduction of the lipid-lowering effect of statins. ${ }^{15-19}$ In addition, genetic variants of the drugmetabolizing enzyme such as cytochrome P450 $3 A 4$ (CYP3A4) and cytochrome P450 $3 A 5$ (CYP3A5) gene polymorphisms have been suggested that related to the lipid-lowering efficacy of statins. ${ }^{20,21}$ However, the influences of genetic polymorphisms have been inconsistently replicated, and also genetic variations are related to ethnicity; the interethnic differences can be a noteworthy factor in drug response. ${ }^{22,23}$ The study of Na Nakorn et al reported that the allele frequencies of the c.388A $>\mathrm{G}$ variants in the $S L C O 1 B 1$ gene were different among the Thai population and Asian Indians and Caucasians. ${ }^{24}$ The Affymetrix Drug Metabolizing Enzyme and Transporters (DMET) microarray platform has been demonstrated that it is valuable in studying interethnic in drug response. ${ }^{25}$ The Affymetrix DMET platform is a useful tool for simultaneous genotyping of a large number of genetic polymorphisms, which investigates a total of 1936 genetic markers (1931 SNPs and 5 CNVs), over 231 pharmacokinetics-related genes. A broad approach in evaluating the influence of multiple DMET gene variations on the lipid-lowering efficacy of statins may offer a better understanding of their etiological mechanisms. However, there have not been any association studies between genetic variants of DMET genes and the lipid-lowering response of statin in the Thai population. The objective of this study was to investigate the association between genetic polymorphisms of the DMET genes and lipid-lowering response of statin in the Thai population by using the Affymetrix DMET Plus genotyping microarrays.

\section{Materials and Methods}

\section{Eligible Patients}

Seventy-nine patients subjected to treatment with statins were recruited from the Faculty of Medicine Ramathibodi Hospital, Mahidol University, Bangkok, Thailand. For this retrospective cohort study, patients (20-80 years old) received statins for at least 3 months. Serum lipid profile was measured at baseline (the first time of sampling) and repeated after 4-month on a statin regimen. The exclusion criteria were (1) patients with liver disease, renal disease, familial hypercholesterolemia, uncontrolled thyroid disease, and/or malignant diseases; (2) patients receiving concomitant medications that could potentially affect the lipid profile and pharmacokinetics of statins; (3) adjusted dose during treatment; and (4) non-compliant patients. The study was approved by the Ramathibodi Ethics Committee (MURA2019/ 735). All methods were carried out per the Declaration of Helsinki.

\section{DMET Plus Array and Quality Control}

Genomic DNA was extracted from EDTA whole blood using the MagNA Pure automated extraction system (Roche Applied Science, Penzberg, Germany). The quality and quantity of genomic DNA were measured by using NanoDrop Spectrophotometer (Thermo Fisher Scientific, Wilmington, DE, USA). 
Genotype analysis using the DMET Plus platform is based on an efficient and comprehensive molecular inversion probe (MIP) technology (Affymetrix Inc., Santa Clara, CA, USA). The Affymetrix DMET ${ }^{\mathrm{TM}}$ Plus microarray enables individuals to test a total of 1936 genetic markers including 1931 SNPs and 5 copy number variations (CNVs) across 231 genes having functional significance in drug pharmacokinetics. ${ }^{26}$ The PCR products from MIP technology were fragmented by fragmentation enzyme and then hybridized on DMET microarray. ${ }^{27,28}$ Affymetrix fluidic stations were used to wash and stain the DMET arrays. DNA samples on the DMET Plus array were scanned with the Affymetrix GeneChip R Scanner3000 (AffymetrixInc, Santa Clara, CA, USA). The genotyping profiles of DMET SNPs were generated by DMET ${ }^{\mathrm{TM}}$ Console Software ${ }^{\circledR}$ (Version 1.3; Affymetrix Inc.). Quality control of genotyping data was checked by using the R package "GenABEL" and Haploview software. ${ }^{29}$ Individuals with a $<95 \%$ genotyping call rate were excluded. SNPs with minor allele frequency (MAF) $<0.05$, SNPs with more than $5 \%$ missing genotypes, a deviation from Hardy-Weinberg equilibrium (HWE) at $P>0.05$, and variants on chromosome $\mathrm{X}$ were also excluded.

\section{Statistical Analysis}

The demographic and phenotypic measurements were analyzed using the SPSS (version 22.0). The normal distribution of the data was tested by Kolmogorov-Smirnov test. As the data are not normally distributed, continuous data were reported as the median and interquartile range (Q1-Q3). Association between types of statins and percent changes in lipid profile was analyzed using Kruskal-Wallis Test. $P$-value $<0.05$ was considered significant. Multidimensional scaling (MDS) plot and association analysis between DMET markers and percent changes in lipid profile were estimated using CochranArmitage test for trends in the GenABEL package of $\mathrm{R}$ version 3.1.1. ${ }^{30}$ The $P$-value adjusted after Bonferroni correction was set at $2.5 \times 10^{-5}$. However, as an exploratory study, genetic markers were selected as prominent ones in the association analysis with a $P$-value less than 0.01 , corrected for possible inflation due to some degree of stratification. Pairwise linkage disequilibrium analysis was performed using Haploview 4.2.

\section{Results}

\section{Demographic Data and Clinical Characteristics}

Seventy-nine participants, the sample group, were mostly female $(\mathrm{n}=48 ; 60.80 \%)$. The median age of patients was 63.00 years (IQR: $55.00-72.00)$. Simvastatin was the most prescribed (81.01\%) followed by atorvastatin (12.66\%), and pitavastatin (6.33\%), respectively. For statins dosing, the percentage of the patients treated with statins $2 \mathrm{mg} / \mathrm{day}, 10$ $\mathrm{mg} / \mathrm{day}, 20 \mathrm{mg} / \mathrm{day}$, and $40 \mathrm{mg} /$ day were $6.33 \%, 50.63 \%, 27.85 \%$, and $15.19 \%$, respectively. Comorbidity among patients was hypertension (29.11\%) and diabetes mellitus (25.32\%) (Table 1). There was no association between types and doses of statins and percentage change in lipid profile as shown in Table 2.

\section{Association Between DMET SNPs and Lipid-Lowering Responses to Statin}

After filtering 80 samples and 1931 SNP markers from DMET ${ }^{\mathrm{TM}}$ Plus GeneChip microarray platform (Affymetrix Inc., Santa Clara, CA, USA) with the quality control parameters, one sample was removed due to low call rate $(<95 \%), 194$ (10.04\%) markers were excluded because of an overall call rate of less than 95\%. For the remaining 1737 markers, 1232 (70.92\%) markers were removed owing to minor allele frequency (MAF) less than 5\%. Also, 46 markers on the $\mathrm{X}$ chromosome and 31 markers with $P$-value $<0.05$ in HWE were excluded as well. As a result, only 79 samples $(98.75 \%)$ and 419 (21.69\%) markers of 1931 markers were included for final analysis. A multidimensional plot did not show any clear clusters, which means that no population stratification existsand also there were no differences in allele frequencies among the studied population (see Supplementary Figure S1).

The DMET SNPs showed significant associations with the percentage change in lipid profile with the $P$-value $<0.01$. The top significant SNPs associated with lipid profile were shown in Table 3 and Figure 1. Of all the DMET SNPs, five SNPs of three genes (solute carrier organic anion transporter family member $1 B 3$ (SLCO1B3); c.334G > T (rs4149117), c.699A > G (rs7311358), and c.1557G > A (rs2053098), quinolinate phosphoribosyltransferase (QPRT); $\mathrm{T}>\mathrm{G}$ (rs13331798), and solute carrier family 10 member 2 (SLC10A2); c.511G $>\mathrm{T}$ ( $\mathrm{rs} 188096)$ were found to be associated with the percentage change in LDL-cholesterol after 4 months of statin treatment $(P$-value $<0.01)$ (Table 3 and 
Table I Demographic Data and Clinical Characteristics of Study Subjects $(n=79)$

\begin{tabular}{|c|c|}
\hline Parameters & Median (IQR) \\
\hline Gender (Male/Female), n (\%) & $31(39.20) / 48(60.80)$ \\
\hline Age, years & $63.00(55.00-72.00)$ \\
\hline \multicolumn{2}{|l|}{ Types of statins, n (\%) } \\
\hline Simvastatin & $64(81.01)$ \\
\hline Atorvastatin & $10(12.66)$ \\
\hline Pitavastatin & $5(6.33)$ \\
\hline \multicolumn{2}{|l|}{ Doses of statins, $\mathrm{n}(\%)$} \\
\hline $2 \mathrm{mg}$ & $5(6.33)$ \\
\hline $10 \mathrm{mg}$ & $40(50.63)$ \\
\hline $20 \mathrm{mg}$ & $22(27.85)$ \\
\hline $40 \mathrm{mg}$ & $12(15.19)$ \\
\hline Body mass index $(\mathrm{BMI})\left(\mathrm{kg} / \mathrm{m}^{2}\right)$ & $26.60(24.50-30.80)$ \\
\hline \multicolumn{2}{|l|}{ Blood pressure } \\
\hline Systolic blood pressure $(\mathrm{mmHg})$ & $140.00(126.00-154.00)$ \\
\hline Diastolic blood pressure $(\mathrm{mmHg})$ & $77.00(69.00-90.00)$ \\
\hline \multicolumn{2}{|l|}{ Lipid levels (baseline) } \\
\hline Total cholesterol (TC), mg/dl & $179.00(\mid 56.00-203.00)$ \\
\hline LDL cholesterol (LDL-C), mg/dl & $104.00(89.0-124.00)$ \\
\hline HDL cholesterol (HDL-C), mg/dl & $44.00(37.00-47.00)$ \\
\hline Triglyceride (TG), mg/dl & $195.00(161.00-239.00)$ \\
\hline \multicolumn{2}{|l|}{ Lipid levels (after 4 months) } \\
\hline Total cholesterol (TC), mg/dl & $170.00(\mid 47.00-200.00)$ \\
\hline LDL cholesterol (LDL-C), mg/dl & $103.00(82.00-125.00)$ \\
\hline HDL cholesterol (HDL-C), mg/dl & $43.00(37.00-49.00)$ \\
\hline Triglyceride (TG), mg/dl & $172.00(\mid 40.00-205.00)$ \\
\hline \multicolumn{2}{|l|}{ Percentage change $(\% \Delta)$} \\
\hline Total cholesterol (TC) & $-4.49(-13.24-4.17)$ \\
\hline LDL cholesterol (LDL-C) & $-4.8 \mid(-15.3 \mid-9.57)$ \\
\hline HDL cholesterol (HDL-C) & $0.00(-8.00-5.88)$ \\
\hline Triglyceride (TG) & $-10.49(-29.52-11.97)$ \\
\hline \multicolumn{2}{|l|}{ Comorbidity } \\
\hline Hypertension, n (\%) & $23(29.11)$ \\
\hline Diabetes mellitus, n (\%) & $20(25.32)$ \\
\hline
\end{tabular}

Abbreviation: IQR, interquartile range.

Figure 1A). The percentage change in HDL-cholesterol after 4 months of statin treatment correlated significantly with the cytochrome P450 $7 A 1$ (CYP7A1); -1744G $>\mathrm{A}$ (rs12542233) gene polymorphism ( $P$-value $<0.01)$ as shown in Table 3 and Figure 1B. The top significant DMET SNPs associated with the percentage change in total cholesterol after 4 months of 
Table 2 Association Between Types and Doses of Statins and Percentage Change in Lipid Profile After 4 Months of Statin Treatment $(\mathrm{n}=79)$

\begin{tabular}{|c|c|c|c|}
\hline Statin Response & Types and Doses of Statins & Median (IQR) & $P$-value \\
\hline Percentage change in LDL-cholesterol & $\begin{array}{l}\text { Simvastatin } 10 \mathrm{mg} \\
\text { Simvastatin } 20 \mathrm{mg} \\
\text { Simvastatin } 40 \mathrm{mg} \\
\text { Atorvastatin } 20 \mathrm{mg} \\
\text { Atorvastatin } 40 \mathrm{mg} \\
\text { Pitavastatin } 2 \mathrm{mg}\end{array}$ & $\begin{array}{c}-5.13(-17.14-7.32) \\
-5.26(-17.09-13.33) \\
-4.81(-5.91-33.33) \\
-3.50(-7.00-0.00) \\
-5.77(-12.13-3.61) \\
7.14(1.47-40.62)\end{array}$ & 0.56 \\
\hline Percentage change in HDL-cholesterol & $\begin{array}{l}\text { Simvastatin } 10 \mathrm{mg} \\
\text { Simvastatin } 20 \mathrm{mg} \\
\text { Simvastatin } 40 \mathrm{mg} \\
\text { Atorvastatin } 20 \mathrm{mg} \\
\text { Atorvastatin } 40 \mathrm{mg} \\
\text { Pitavastatin } 2 \mathrm{mg}\end{array}$ & $\begin{array}{c}2.27(-5.56-8.11) \\
-2.13(-8.16-3.12) \\
-2.13(-2.27-5.17) \\
3.85(-10.71-18.42) \\
-6.67(-15.56-4.26) \\
0.00(-3.85-14.28)\end{array}$ & 0.84 \\
\hline Percentage change in total cholesterol & $\begin{array}{l}\text { Simvastatin } 10 \mathrm{mg} \\
\text { Simvastatin } 20 \mathrm{mg} \\
\text { Simvastatin } 40 \mathrm{mg} \\
\text { Atorvastatin } 20 \mathrm{mg} \\
\text { Atorvastatin } 40 \mathrm{mg} \\
\text { Pitavastatin } 2 \mathrm{mg}\end{array}$ & $\begin{array}{l}-5.12(-14.74-2.13) \\
-7.65(-15.73-4.76) \\
-5.59(-8.90-25.45) \\
-6.56(-9.20-3.92) \\
-1.50(-4.91-4.27) \\
13.79(2.32-24.70)\end{array}$ & 0.55 \\
\hline Percentage change in triglyceride & $\begin{array}{l}\text { Simvastatin } 10 \mathrm{mg} \\
\text { Simvastatin } 20 \mathrm{mg} \\
\text { Simvastatin } 40 \mathrm{mg} \\
\text { Atorvastatin } 20 \mathrm{mg} \\
\text { Atorvastatin } 40 \mathrm{mg} \\
\text { Pitavastatin } 2 \mathrm{mg}\end{array}$ & $\begin{array}{c}-9.63(-30.19-18.06) \\
-10.49(-25.53-9.36) \\
-18.24(-20.54-11.27) \\
-21.29(-39.75-2.83) \\
11.62(-21.14-23.44) \\
0.00(-14.72-8.70)\end{array}$ & 0.82 \\
\hline
\end{tabular}

Abbreviation: IQR, interquartile range.

statin treatment were shown in Table 3 and Figure 1C. The analysis showed seven SNPs of three genes (SLCO1B3; c.334G > T (rs4149117), c.699A > G (rs7311358), and c.1557G > A (rs2053098), sulfotransferase family 1E member 1 (SULT1E1); c. -64C > T (rs3736599) and c.-9-469A >G (rs3822172), and ATP Binding Cassette Subfamily B Member 11 ( $A B C B 11) ; c .-12519 C>T$ (rs4148768) and c. $-8583 \mathrm{G}>\mathrm{A}$ (rs3770603) were found to be significantly associated with the percentage change in total cholesterol after 4 months of statin treatment $(P$-value $<0.01)$. Among DMET SNPs, the ATP Binding Cassette Subfamily B Member 4 (ABCB4); c.711A >T (rs2109505) gene polymorphism presented a significant association with percent change in triglyceride after 4 months of statin treatment $(P<0.01)$ (Table 3 and Figure 1D).

\section{Linkage Disequilibrium Analysis}

Linkage disequilibrium analysis of seven SNPs across three genes is shown in Figure 2. Strong linkage disequilibria (D'values >0.9) were observed between SLCO1B3 rs4149117, rs7311358, and rs2053098; $A B C B 11$ rs4148768, and rs3770603; and SULT1E1 rs3736599 and rs3822172.

\section{Discussion}

In the present study, SNPs in drug-metabolizing enzymes and transporter genes associated with lipid-lowering response to statin were identified. The result exhibited that five SNPs; three SLCO1B3 variants (rs4149117, rs7311358, and rs2053098), one $Q P R T$ variant (rs13331798), and one SLC10A2 variant (rs188096) were significantly associated with LDL-cholesterol-lowering response. One SNP of the CYP7A1 gene (rs12542233) was significantly associated with HDLcholesterol-lowering response. Seven SNPs; three SLCO1B3 variants (rs4149117, rs7311358, and rs2053098), two 
Table 3 Top SNPs Associated with Percentage Change in Lipid Profile After 4 Months of Statin Treatment $(n=79)$

\begin{tabular}{|c|c|c|c|c|}
\hline Statin Response & SNP rsID & Marker Name & Chromosome & $P$-values \\
\hline \multirow[t]{5}{*}{ Percentage change in LDL-cholesterol } & rs4l49II7 & SLCOIB3 C.334G>T & 12 & 0.0002 \\
\hline & rs7311358 & SLCOIB3 C.699A>G & 12 & 0.0002 \\
\hline & rs2053098 & SLCOIB3 C. I557G>A & 12 & 0.0002 \\
\hline & rs|333|798 & $Q P R T \_T>G$ & 16 & 0.0059 \\
\hline & rs 188096 & $S L C I 0 A 2$ C.5IIG>T & 13 & 0.0068 \\
\hline Percentage change in HDL-cholesterol & rs 12542233 & CYP7AI_-I 744G>A & 8 & 0.0081 \\
\hline \multirow[t]{7}{*}{ Percentage change in total cholesterol } & rs4149II7 & SLCOIB3 C.334G>T & 12 & 0.0015 \\
\hline & rs731I358 & SLCOIB3 C.699A>G & 12 & 0.0015 \\
\hline & rs2053098 & SLCOIB3 C. $1557 \mathrm{G}>A$ & 12 & 0.0015 \\
\hline & rs3736599 & SULTIEI c. $-64 C>T$ & 4 & 0.0095 \\
\hline & rs3822172 & SULTIEI_c.-9-469A >G & 4 & 0.0068 \\
\hline & rs4l48768 & $A B C B \mid I \_C .-12519 C>T$ & 2 & 0.0064 \\
\hline & rs3770603 & $A B C B \mid I$ C. $-8583 G>A$ & 2 & 0.0077 \\
\hline Percentage change in triglyceride & rs2109505 & $A B C B 4$ c.7| IA>T & 7 & 0.0064 \\
\hline
\end{tabular}

SULT1E1 variants (rs3736599 and rs3822172), and two $A B C B 11$ variants (rs4148768 and rs3770603) were significantly associated with the total cholesterol-lowering response. One SNP of the $A B C B 4$ gene (rs2109505) was significantly associated with triglyceride-lowering response.

Regarding the effect of drug transporter genes, $S L C O 1 B 3$ and $S L C 1 O A 2$ are the hepatic influx transporters that are involved in statins clearance and impact lipid concentration. The SLCO1B3 gene is translated into organic anion transporting polypeptide 1B3 (OATP1B3) protein, which is the hepatic uptake transporters involved in statins' clearance. In an in vitro study, HeLa cells transfected with SLCO1B3 (c.334G) and (c.699A) haplotype presented a decrease in statin uptake. ${ }^{31}$ Many SNPs of the SLCO1B3 gene including SNPs 334T $>$ G (Ser112Ala), 699A $>$ G (Met233Ile), 1564C $>\mathrm{T}$ (Gly522Cys), and 1564C $>\mathrm{T}$ (Gly522Cys) have been analyzed by Letschert et al about functional consequences on protein localization and transport characteristics. ${ }^{32}$ A previous study in the Korean population reported that SLCO1B3 c.334 $\mathrm{T}>\mathrm{G}$ was associated with higher statin drug concentrations. ${ }^{33}$ The present study found SLCO1B3-c.334T $>\mathrm{G}$ (rs4149117) encoding OATP1B3- S112A, SLCO1B3-c.699A $>$ G (rs7311358) encoding OATP1B3- M233I, and SLCO1B3-c.1557G $>$ A (rs2053098) encoding OATP1B3- A519A strongly associated with lipid-lowering responses to statin. There are strong linkage disequilibria (D'-values $>0.9$ ) among rs4149117, rs7311358, and rs2053098 of the SLCO1B3 gene. It might be possible that SLCO1B3 (c.334G > T, c.699A > G, and c.1557G > A) gene polymorphisms may influence statin levels and lipid plasma concentration in the patient treated with a statin. Concerning the SLC10A2 gene, it is an apical sodium-dependent bile salt transporter (ASBT) or the ileal bile acid transporter which mediates the intestinal absorption of bile salt. As revealed by systematic screening and functional characterization of human SLC10A2 alleles, the functionally disruptive SNPs are rare. ${ }^{34,35}$ The genetic polymorphism of the SLC10A2 gene is affected by ethnicity. Among genetic variations of the SLC10A2 gene, the most prevalent was the functionally silent $511 \mathrm{G}>\mathrm{T}$ (Ala171Ser), found in European American (6\%), Chinese American (4.5\%), Hispanic American (3.9\%), and African American (1.7\%) populations. ${ }^{34}$ Our study revealed that SNP SLC10A2-c.511G $>$ T (rs188096) was significantly associated with the lipid-lowering response to statin. The underlying molecular mechanism of $S L C 10 A 2$ gene polymorphisms and lipid-lowering responses to statin should be investigated in further study to reveal the linking molecular pathway. 
A

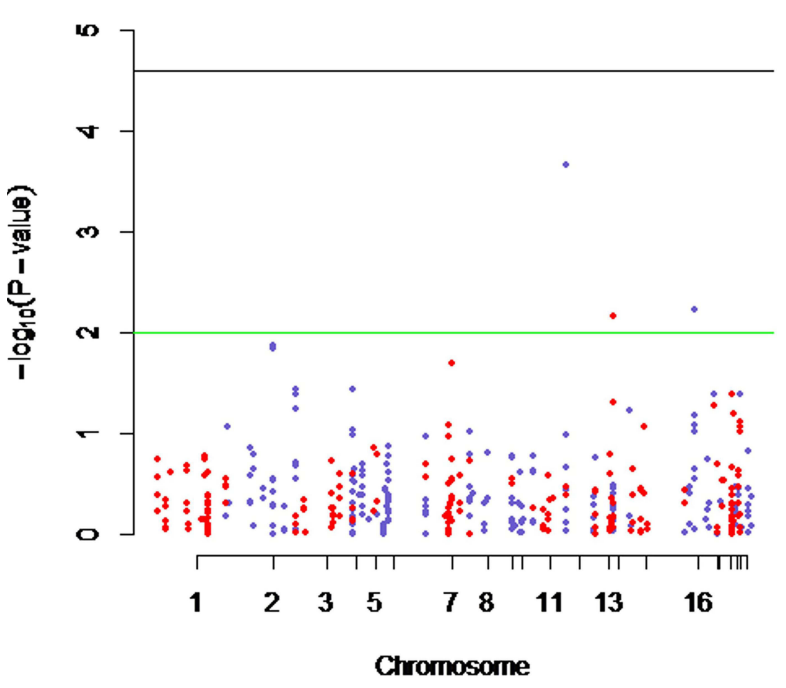

C

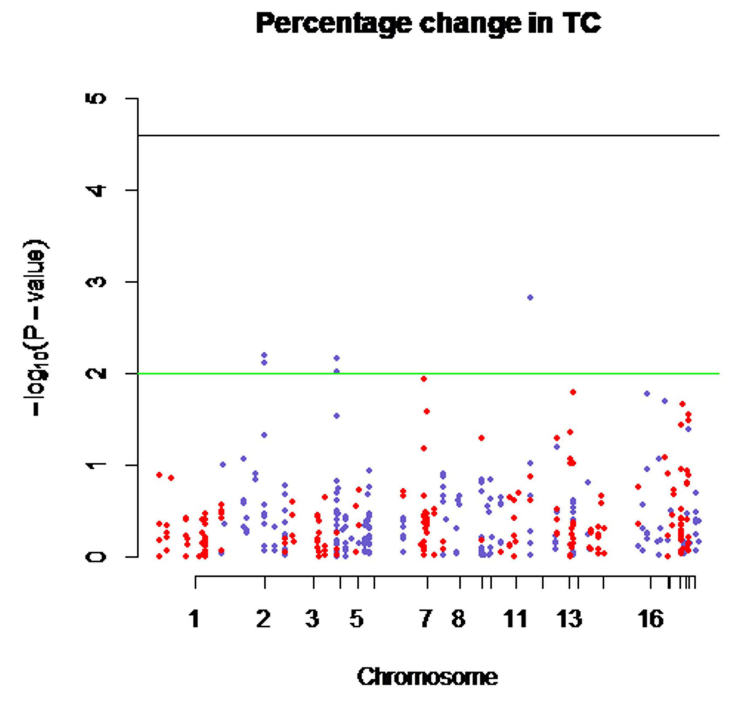

B Percentage change in HDL-C

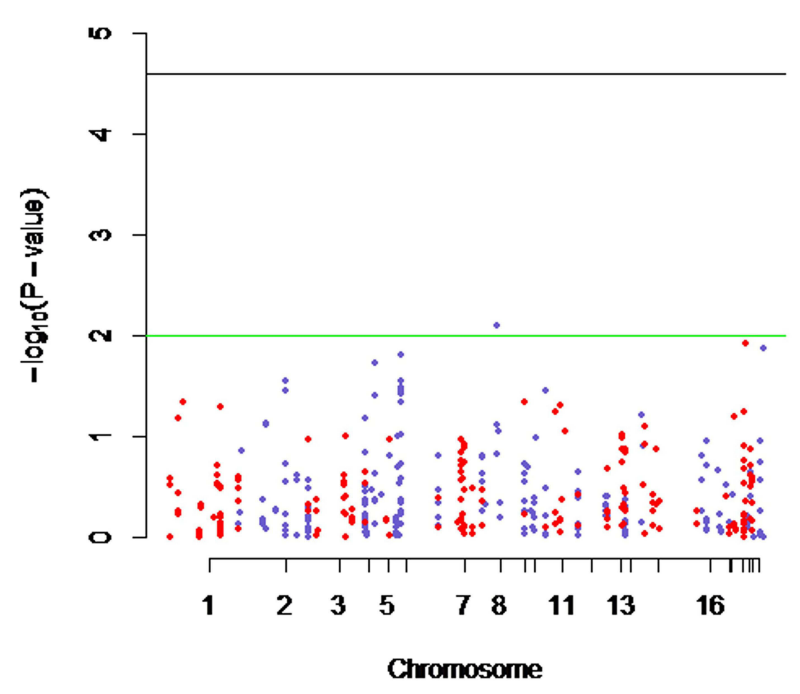

D Percentage change in TG

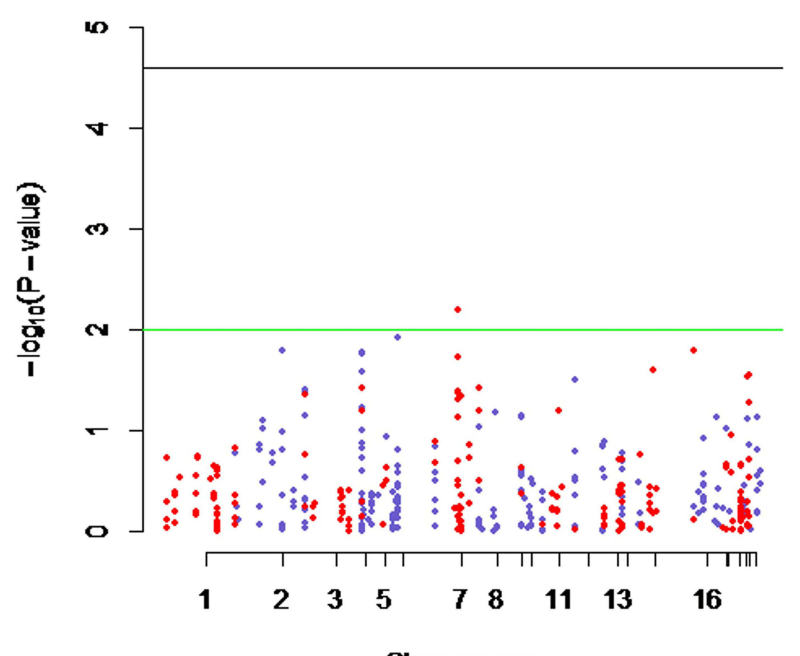

Chromosome

Figure I Manhattan plot of - $\log 10$ (P-values) from Cochran-Armitage test for trends of 419 single nucleotide polymorphisms (SNPs) in drug-metabolizing enzyme and transporter (DMET) genes and percentage changes in LDL-cholesterol (LDL-C), HDL-cholesterol (HDL-C), total cholesterol (TC), and triglyceride (TG) levels. The X-axis indicates chromosome number and Y-axis indicates - $\log 1 \mathrm{OP}$-values. The green line indicates a $P$-value of 0.01 . The black line indicates a $P$-value of $2.5 \times 10^{-5}$. (A) Manhattan plot demonstrates the significant DMET SNPs associated with the percentage change in LDL-cholesterol (B) Manhattan plot demonstrates the significant DMET SNPs associated with the percentage change in HDL-cholesterol (C) Manhattan plot demonstrates the significant DMET SNPs associated with the percentage change in total cholesterol and (D) Manhattan plot demonstrates the significant DMET SNPs associated with the percentage change in triglyceride.

Concerning efflux transporter genes, the present study found that $A B C B 4$ and $A B C B 11$ transporter genes polymorphisms were significantly associated with lipid-lowering responses to statin. $A B C B 4$ and $A B C B 11$ transporters are both located in the liver and implicated in canalicular bile formation and lipid secretion. $A B C B 4$ gene, also known as multidrug resistance protein 3 (MDR3), is a membrane-associated transport protein almost exclusively expressed in the liver ${ }^{36,37}$ that elaborated in biliary phospholipid secretion. ${ }^{38}$ Biliary phospholipids play a key role in solubilizing cholesterol. $A B C B 11$ gene is translated to the bile salt export pump (BSEP) that is involved in most of the bile salt transport from hepatocytes into the bile canalicular lumen. ${ }^{39}$ Hirano et al suggested that BSEP might be elaborated in the biliary excretion of statin in both rats and humans. ${ }^{40} A B C B 4$ and $A B C B 11$ gene polymorphisms have been reported to affect the composition of bile and were associated with cholestasis and cholelithiasis. ${ }^{41-43}$ Hypercholesterolemia is a 


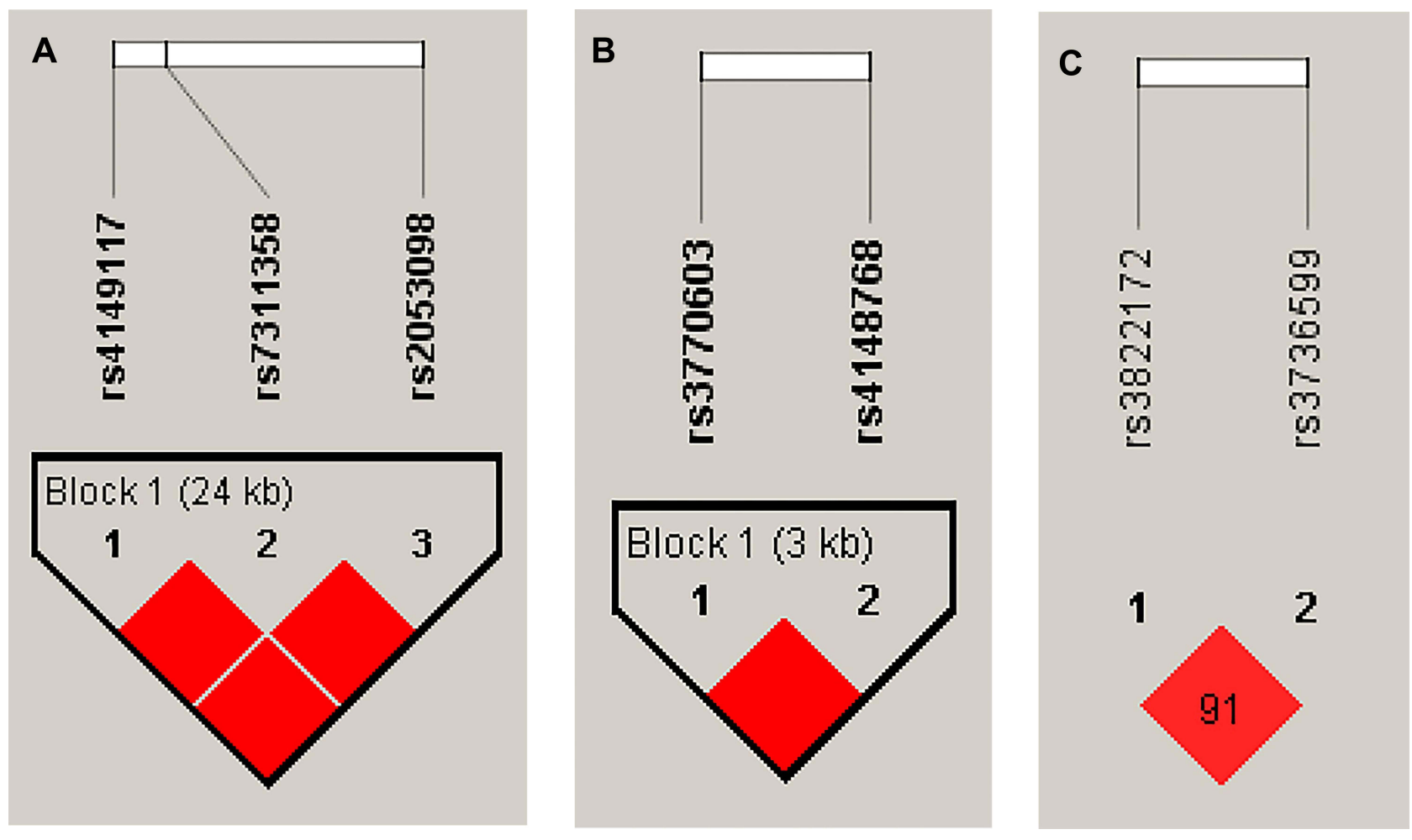

Figure 2 Haploview linkage disequilibrium map of SNPs across DMET genes: (A) SLCOIB3; (B) ABCBII; and (C) SULTIEI. Pairwise linkage disequilibrium (D') values are given in blocks for each SNP combination. Empty dark red blocks indicate D' values of I.0.

common feature of cholestasis because its metabolic degradation and excretion are impaired. The present study identified one novel polymorphism in $A B C B 4$ (rs2109505) and two novel polymorphisms in $A B C B 11$ (rs4148768 and rs3770603) strongly associated with the lipid-lowering response to statin. Taken together with other data, it might be possible that the genetic variations in $A B C B 11$ and $A B C B 4$ genes may cause the altered protein function of MDR3 and BSEP leading to alterations in the statins effluxion rate and biliary excretion, which is impact on cholesterol levels.

Cytochrome P450 (CYP) enzyme, cholesterol 7 -hydroxylase (CYP7A1), the rate-limiting enzyme in bile acid production is one of the most promising candidate genes for the determination of individual pharmacological lowering of plasma cholesterol. CYP7A1 is the initial and rate-limiting step for the removal of cholesterol into bile and plays a vital role in maintaining cholesterol homeostasis. ${ }^{44,45}$ Many studies have demonstrated that CYP7A1 variants affect the LDL cholesterol-lowering response to statin. ${ }^{46-49}$ A meta-analysis reported that the A-204C (CYP7A1) polymorphism was related to the level of total cholesterol and the lipid-lowering efficacy of statin treatment. ${ }^{50}$ In this study, we found a significant association between a novel CYP7A1 polymorphism rs12542233 and reductions of HDL cholesterol response to a statin in Thai patients with dyslipidemia. In further studies, the mechanistic functional effects of CYP7A1 (rs12542233) polymorphism should be examined to reveal the molecular pathways.

Other top SNPs associated with the lipid-lowering response to statin are the SNP in the QPRT gene and SNPs in the SULT1E1 gene. The QPRT or quinolinate phosphoribosyltransferase is an important enzyme in the synthesis of de novo nicotinamide adenine dinucleotide $(\mathrm{NAD}+) .{ }^{51}$ The increased NAD+ levels were found to stimulate sirtuin 1 (SIRT1) activity, leading to activate the peroxisome proliferator-activated receptor $\gamma$ coactivator $1-\alpha$ (PGC-1 $\alpha$ ), an important regulator of mitochondrial biogenesis thereby enhancing mitochondrial fatty acid oxidation and reducing triglyceride synthesis. An activated SIRT1 enhances ABCA1 expression by stimulating LXR resulting in increased circulating HDL particles. ${ }^{52}$ Moreover, the SIRT1 also reduces SREBP-mediated fatty acid, triglyceride, and cholesterol synthesis by destabilizing SREBPs. ${ }^{52-54}$ The study from Shin et al reported that regulation of the key enzymes in the TryptophanNAD+ pathway, including QPRT, was associated with PPAR-alpha directly or indirectly. ${ }^{55}$ Statins are known to be an activator of PPAR-alpha promoter and mRNA expression. ${ }^{56}$ It might be hypothesized that statins may influence 
cholesterol levels by indirectly promoting QPRT activity. Our study found that SNP $Q P R T$ T $>$ G (rs13331798) was significantly associated with lipid-lowering response to statin. However, this SNP is not in the coding region and does not have direct interactions with lipid-lowering response to statin. Also, the mechanisms underlying $Q P R T$ gene polymorphisms related to lipid-lowering response to statin has not been elucidated, and further study is needed to discover mechanisms underlying the relationship between the QPRT gene and lipid-lowering response to statin.

Regarding, the sulfotransferase family $1 E$ member 1 (SULT1E1) encoding SULT1E1 enzymes. Sulfotransferases (SULTs) play critical roles in sulfating estrogens, thyroid hormones, bile acids, and other xenobiotics. The previous study showed that SULT1E1 regulates inflammatory response and lipid metabolism by upregulating Peroxisome proliferatoractivated receptor- $\alpha$ (PPAR- $\alpha),{ }^{57}$ which is a nuclear receptor that regulates lipid and glucose metabolism. ${ }^{58}$ Yano et al demonstrated that statins can activate PPAR via the pathway of Cyclooxygenase-2 (COX-2), ${ }^{59}$ which is a key enzyme in fatty acid metabolism. Our study found that the novel SNPs SULT1E1 c. -64C>T (rs3736599) and SULT1E1_c.-9$469 \mathrm{~A}>\mathrm{G}$ (rs3822172) associated with lipid-lowering response to statin. Nevertheless, the relationship of SULT1E1 with lipid-lowering response to statin has not been clearly stated in the studies conducted to date. In the future, it is necessary to investigate the mechanistic functional effects of SULT1E1 gene polymorphism on statin responses to reveal the linking molecular pathways.

In addition to pharmacokinetics genes of statin, the polymorphisms of pharmacodynamics genes such as CETP, ApoE, lipoprotein lipase ( $L P L)$, low-density lipoprotein receptor ( $L D L R)$, and $L X R$ also influence susceptibility to dyslipidemia and statin response. ${ }^{60-62}$ Moreover, $L D L R$ and $L X R$ genes polymorphisms were found to be associated with variations in blood pressure, ${ }^{63}$ which is one of the risk factors for cardiovascular disease. Further studies are needed to investigate the impact of the genetic variations in the pharmacodynamic pathway on statin response in the Thai population.

There are several limitations of our study. First, many SNP markers were removed after the QC process because of low minor allele frequency (MAF < 0.05) leading to a missing of the rare genetic variants which may relate to the statin responses. However, selected SNPs (MAF $\geq 0.05$ ) could avoid the false-positive results. Second, the relatively small sample size might have limited the power to examine the relationship between DMET gene polymorphisms and the lipidlowering response to statins. Third, lack of validation of the significant DMET SNPs and association of lipid-lowering response to statin. The findings should be verified in an independent cohort to avoid the risk of false-positive findings. In addition, some of the SNPs with a suggestive association in this study were not shown to play a vital role in the pharmacokinetic pathway of statin. The evidence of pathway linkup must be established in future studies. Fourth, the changes in serum lipid profile may not be seen clearly due to the short follow-up period. Finally, our study is a retrospective study. The serum lipid profile of the patients at the initiation of statin treatment and other factors involved in statin response such as dietary and lifestyle were not obtainable. Future studies need to design as prospective studies for minimizing the limitations and improving the analysis performance.

\section{Conclusion}

In summary, this study genotyped multiple polymorphisms in genes related to phase I and II drug metabolism enzymes and drug transporters and assessed the relationship between DMET SNPs and lipid-lowering response to a statin in the Thai population. Our findings provide a pharmacogenomic approach to further investigate the DMET gene polymorphisms, which impact lipid-lowering response to statin. We suggest further validation of the findings in different cohorts with prospective study design and larger sample sizes. In addition, the evidence of pathway linkup between significant DMET SNPs and lipid-lowering response to statin needs to be established in future studies.

\section{Ethics Statement}

This study was approved by the Ethics Committee of the Faculty of Medicine, Ramathibodi Hospital, Mahidol University (MURA2019/735). Informed Consent was waived due to the research use specimens leftover from clinical care at the Division of Clinical Chemistry, Department of Pathology, Faculty of Medicine, Ramathibodi Hospital, Mahidol University, Bangkok, Thailand. The specimens were not collected specifically for the proposed research, and no additional specimen was collected for this research. Also, the analysis used anonymous clinical data. 


\section{Acknowledgments}

The authors thank all the staff of the Division of Pharmacogenomics and Personalized Medicine and Division of Clinical Chemistry, Department of Pathology Faculty of Medicine Ramathibodi Hospital, Mahidol University, including all the patients who participated in the study.

\section{Funding}

This research was funded by (1) the Special Task Force for Activating Research (STAR), Rachadapisek Somphot Fund, Chulalongkorn University (STF6300237001-1), (2) Thailand Center of Excellence for Life Sciences (TCELS) (TC 15/ 63), and (3) the Faculty of Medicine Ramathibodi Hospital, Mahidol University.

\section{Disclosure}

The authors have no conflicts of interest to declare.

\section{References}

1. Zhao J, Kelly M, Bain C, Seubsman SA, Sleigh A. Risk factors for cardiovascular disease mortality among 86866 members of the Thai Cohort Study, 2005-2010. Glob J Health Sci. 2015;7(1):107-114. doi:10.5539/gjhs.v7n1p107

2. Taylor F, Huffman MD, Macedo AF, et al. Statins for the primary prevention of cardiovascular disease. Cochrane Database Syst Rev. 2013;2013(1): Cd004816.

3. Baigent C, Blackwell L, Emberson J, et al. Efficacy and safety of more intensive lowering of LDL cholesterol: a meta-analysis of data from 170,000 participants in 26 randomised trials. Lancet. 2010;376(9753):1670-1681.

4. Gitt AK, Drexel H, Feely J, et al. Persistent lipid abnormalities in statin-treated patients and predictors of LDL-cholesterol goal achievement in clinical practice in Europe and Canada. Eur J Prev Cardiol. 2012;19(2):221-230. doi:10.1177/1741826711400545

5. Reiner Ž, Tedeschi-Reiner E. Prevalence and types of persistent dyslipidemia in patients treated with statins. Croat Med J. 2013;54(4):339-345. doi:10.3325/cmj.2013.54.339

6. Wang Y, Du X, Zhao R, Niu J, Wang H, Li J. Association of APOE polymorphisms with lipid-lowering efficacy of statins in atherosclerotic cardiovascular diseases. Ann Acad Med Singap. 2021;50(6):474-480. doi:10.47102/annals-acadmedsg.2020505

7. Donnelly LA, Palmer CN, Whitley AL, et al. Apolipoprotein E genotypes are associated with lipid-lowering responses to statin treatment in diabetes: a Go-DARTS study. Pharmacogenet Genomics. 2008;18(4):279-287. doi:10.1097/FPC.0b013e3282f60aad

8. Srisawasdi P, Rodcharoen P, Vanavanan S, et al. Association of CETP gene variants with atherogenic dyslipidemia among Thai patients treated with statin. Pharmgenomics Pers Med. 2021;14:1-13.

9. Gu GL, Xu XL, Yang QY, Zeng RL. Effect of CETP polymorphism on atorvastatin lipid-regulating effect and clinical prognosis of patients with coronary heart disease. Med Sci Monit. 2014;20:2824-2829. doi:10.12659/MSM.892711

10. Zhou YF, Zhang J, Li ZX, et al. Association of liver X receptor $\alpha(\mathrm{LXR} \alpha)$ gene polymorphism and coronary heart disease, serum lipids and glucose levels. Lipids Health Dis. 2014;13:34. doi:10.1186/1476-511X-13-34

11. Yang JS, Hao JJ, Wang SS, et al. Association between the LXR $\alpha$ polymorphism and stroke in a Chinese population. Genet Mol Res. 2015;14 (1):1757-1762. doi:10.4238/2015.March.13.2

12. McGraw J, Waller D. Cytochrome P450 variations in different ethnic populations. Expert Opin Drug Metab Toxicol. 2012;8(3):371-382. doi:10.1517/17425255.2012.657626

13. Tomalik-Scharte D, Lazar A, Fuhr U, Kirchheiner J. The clinical role of genetic polymorphisms in drug-metabolizing enzymes. Pharmacogenomics J. 2008;8(1):4-15. doi:10.1038/sj.tpj.6500462

14. Evrard A, Mbatchi L. Genetic polymorphisms of drug metabolizing enzymes and transporters: the long way from bench to bedside. Curr Top Med Chem. 2012;12(15):1720-1729. doi:10.2174/156802612803531388

15. Peters BJ, Rodin AS, Klungel OH, et al. Pharmacogenetic interactions between ABCB1 and SLCO1B1 tagging SNPs and the effectiveness of statins in the prevention of myocardial infarction. Pharmacogenomics. 2010;11(8):1065-1076. doi:10.2217/pgs.10.81

16. Romaine SP, Bailey KM, Hall AS, Balmforth AJ. The influence of SLCO1B1 (OATP1B1) gene polymorphisms on response to statin therapy. Pharmacogenomics J. 2010;10(1):1-11. doi:10.1038/tpj.2009.54

17. Elsby R, Hilgendorf C, Fenner K. Understanding the critical disposition pathways of statins to assess drug-drug interaction risk during drug development: it's not just about OATP1B1. Clin Pharmacol Ther. 2012;92(5):584-598. doi:10.1038/clpt.2012.163

18. Chasman DI, Giulianini F, MacFadyen J, Barratt BJ, Nyberg F, Ridker PM. Genetic determinants of statin-induced low-density lipoprotein cholesterol reduction: the justification for the use of statins in prevention: an intervention trial evaluating rosuvastatin (Jupiter) trial. Circ Cardiovasc Genet. 2012;5(2):257-264. doi:10.1161/CIRCGENETICS.111.961144

19. Fiegenbaum M, da Silveira FR, Van der Sand CR, et al. The role of common variants of ABCB1, CYP3A4, and CYP3A5 genes in lipid-lowering efficacy and safety of simvastatin treatment. Clin Pharmacol Ther. 2005;78(5):551-558. doi:10.1016/j.clpt.2005.08.003

20. Willrich MA, Hirata MH, Genvigir FD, et al. CYP3A53A allele is associated with reduced lowering-lipid response to atorvastatin in individuals with hypercholesterolemia. Clin Chim Acta. 2008;398(1-2):15-20. doi:10.1016/j.cca.2008.07.032

21. Gao Y, Zhang LR, Fu Q. CYP3A4*1G polymorphism is associated with lipid-lowering efficacy of atorvastatin but not of simvastatin. Eur J Clin Pharmacol. 2008;64(9):877-882. doi:10.1007/s00228-008-0502-x

22. Chen ML. Ethnic or racial differences revisited: impact of dosage regimen and dosage form on pharmacokinetics and pharmacodynamics. Clin Pharmacokinet. 2006;45(10):957-964. doi:10.2165/00003088-200645100-00001 
23. Rosenberg NA, Pritchard JK, Weber JL, et al. Genetic structure of human populations. Science. 2002;298(5602):2381-2385. doi:10.1126/ science. 1078311

24. Na Nakorn C, Waisayarat J, Dejthevaporn C, Srisawasdi P, Wongwaisayawan S, Sukasem C. Genetic variations and frequencies of the two functional single nucleotide polymorphisms of SLCO1B1 in the Thai population. Front Pharmacol. 2020;11:728. doi:10.3389/fphar.2020.00728

25. Sissung TM, English BC, Venzon D, Figg WD, Deeken JF. Clinical Pharmacology and Pharmacogenetics in a genomics era: the DMET platform. Pharmacogenomics. 2010;11(1):89-103. doi:10.2217/pgs.09.154

26. Arbitrio M, Di Martino MT, Scionti F, et al. DMET ${ }^{\mathrm{TM}}$ (Drug Metabolism Enzymes and Transporters): a pharmacogenomic platform for precision Medicine. Oncotarget. 2016;7(33):54028-54050. doi:10.18632/oncotarget.9927

27. Burmester JK, Sedova M, Shapero MH, Mansfield E. DMET microarray technology for pharmacogenomics-based Personalized Medicine. Methods Mol Biol. 2010;632:99-124.

28. Hardenbol P, Banér J, Jain M, et al. Multiplexed genotyping with sequence-tagged molecular inversion probes. Nat Biotechnol. 2003;21(6):673678. doi:10.1038/nbt821

29. Barrett JC, Fry B, Maller J, Daly MJ. Haploview: analysis and visualization of LD and haplotype maps. Bioinformatics. 2005;21(2):263-265. doi:10.1093/bioinformatics/bth457

30. Aulchenko YS, Ripke S, Isaacs A, van Duijn CM. GenABEL: an R library for genome-wide association analysis. Bioinformatics. 2007;23 (10):1294-1296. doi:10.1093/bioinformatics/btm108

31. Schwarz UI, Meyer Zu Schwabedissen HE, Tirona RG, et al. Identification of novel functional organic anion-transporting polypeptide 1B3 polymorphisms and assessment of substrate specificity. Pharmacogenet Genomics. 2011;21(3):103-114. doi:10.1097/FPC.0b013e328342f5b1

32. Letschert K, Keppler D, König J. Mutations in the SLCO1B3 gene affecting the substrate specificity of the hepatocellular uptake transporter OATP1B3 (OATP8). Pharmacogenetics. 2004;14(7):441-452. doi:10.1097/01.fpc.0000114744.08559.92

33. Woo HI, Kim SR, Huh W, Ko JW, Lee SY. Association of genetic variations with pharmacokinetics and lipid-lowering response to atorvastatin in healthy Korean subjects. Drug Des Devel Ther. 2017;11:1135-1146. doi:10.2147/DDDT.S131487

34. Ho RH, Leake BF, Urquhart BL, Gregor JC, Dawson PA, Kim RB. Functional characterization of genetic variants in the apical sodium-dependent bile acid transporter (ASBT; SLC10A2). J Gastroenterol Hepatol. 2011;26(12):1740-1748. doi:10.1111/j.1440-1746.2011.06805.X

35. Pan W, Song IS, Shin HJ, et al. Genetic polymorphisms in Na+-taurocholate co-transporting polypeptide (NTCP) and ileal apical sodium-dependent bile acid transporter (ASBT) and ethnic comparisons of functional variants of NTCP among Asian populations. Xenobiotica. 2011;41(6):501-510. doi:10.3109/00498254.2011.555567

36. Van der Bliek AM, Baas F, Ten houte de lange T, Kooiman PM, Van der Velde-koerts T, Borst P. The human mdr3 gene encodes a novel P-glycoprotein homologue and gives rise to alternatively spliced mRNAs in liver. EMBO j. 1987;6(11):3325-3331. doi:10.1002/j.1460-2075.1987. tb02653.x

37. Dean M, Rzhetsky A, Allikmets R. The human ATP-binding cassette (ABC) transporter superfamily. Genome Res. 2001;11(7):1156-1166. doi:10.1101/gr. 184901

38. Sticova E, Jirsa M. ABCB4 disease: many faces of one gene deficiency. Ann Hepatol. 2020;19(2):126-133. doi:10.1016/j.aohep.2019.09.010

39. Arrese M, Ananthanarayanan M. The bile salt export pump: molecular properties, function and regulation. Pflugers Arch. 2004;449(2):123-131. doi:10.1007/s00424-004-1311-4

40. Hirano M, Maeda K, Hayashi H, Kusuhara H, Sugiyama Y. Bile salt export pump (BSEP/ABCB11) can transport a nonbile acid substrate, pravastatin. J Pharmacol Exp Ther. 2005;314(2):876-882. doi:10.1124/jpet.105.084830

41. Pauli-Magnus C, Lang T, Meier Y, et al. Sequence analysis of bile salt export pump (ABCB11) and multidrug resistance p-glycoprotein 3 (ABCB4, MDR3) in patients with intrahepatic cholestasis of pregnancy. Pharmacogenetics. 2004;14(2):91-102. doi:10.1097/00008571-200402000-00003

42. Acalovschi M, Tirziu S, Chiorean E, Krawczyk M, Grünhage F, Lammert F. Common variants of ABCB4 and ABCB11 and plasma lipid levels: a study in sib pairs with gallstones, and controls. Lipids. 2009;44(6):521-526. doi:10.1007/s11745-009-3300-Z

43. Marschall HU, Katsika D, Rudling M, Einarsson C. The genetic background of gallstone formation: an update. Biochem Biophys Res Commun. 2010;396(1):58-62. doi:10.1016/j.bbrc.2010.02.143

44. Chiang JY. Bile acids: regulation of synthesis. J Lipid Res. 2009;50(10):1955-1966. doi:10.1194/jlr.R900010-JLR200

45. Hubacek JA, Bobkova D. Role of cholesterol 7alpha-hydroxylase (CYP7A1) in nutrigenetics and pharmacogenetics of cholesterol lowering. Mol Diagn Ther. 2006;10(2):93-100. doi:10.1007/BF03256448

46. Kajinami K, Brousseau ME, Ordovas JM, Schaefer EJ. A promoter polymorphism in cholesterol 7alpha-hydroxylase interacts with apolipoprotein E genotype in the LDL-lowering response to atorvastatin. Atherosclerosis. 2005;180(2):407-415. doi:10.1016/j.atherosclerosis.2004.12.019

47. Poduri A, Khullar M, Bahl A, Sehrawat BS, Sharma Y, Talwar KK. Common variants of HMGCR, CETP, APOAI, ABCB1, CYP3A4, and CYP7A1 genes as predictors of lipid-lowering response to atorvastatin therapy. DNA Cell Biol. 2010;29(10):629-637. doi:10.1089/dna.2009.1008

48. Wei KK, Zhang LR, Zhang Y, Hu XJ. Interactions between CYP7A1 A-204C and ABCG8 C1199A polymorphisms on lipid lowering with atorvastatin. J Clin Pharm Ther. 2011;36(6):725-733. doi:10.1111/j.1365-2710.2010.01227.x

49. Jiang XY, Zhang Q, Chen P, et al. CYP7A1 polymorphism influences the LDL cholesterol-lowering response to atorvastatin. J Clin Pharm Ther. 2012;37(6):719-723. doi:10.1111/j.1365-2710.2012.01372.x

50. Li Q, Hong J, Wu J, et al. The role of common variants of ABCB1 and CYP7A1 genes in serum lipid levels and lipid-lowering efficacy of statin treatment: a meta-analysis. J Clin Lipidol. 2014;8(6):618-629. doi:10.1016/j.jacl.2014.07.010

51. Ullmark T, Montano G, Järvstråt L, et al. Anti-apoptotic quinolinate phosphoribosyltransferase (QPRT) is a target gene of Wilms' tumor gene 1 (WT1) protein in leukemic cells. Biochem Biophys Res Commun. 2017;482(4):802-807. doi:10.1016/j.bbrc.2016.11.114

52. Romani M, Hofer DC, Katsyuba E, Auwerx J. Niacin: an old lipid drug in a new NAD(+) dress. J Lipid Res. 2019;60(4):741-746. doi:10.1194/jlr. S092007

53. Shah SA, Yoon GH, Chung SS, et al. Novel osmotin inhibits SREBP2 via the AdipoR1/AMPK/SIRT1 pathway to improve Alzheimer's disease neuropathological deficits. Mol Psychiatry. 2017;22(3):407-416. doi:10.1038/mp.2016.23

54. Eberlé D, Hegarty B, Bossard P, Ferré P, Foufelle F. SREBP transcription factors: master regulators of lipid homeostasis. Biochimie. 2004;86 (11):839-848. doi:10.1016/j.biochi.2004.09.018

55. Shin M, Ohnishi M, Iguchi S, Sano K, Umezawa C. Peroxisome-proliferator regulates key enzymes of the tryptophan-NAD+ pathway. Toxicol Appl Pharmacol. 1999;158(1):71-80. doi:10.1006/taap.1999.8683 
56. Seo M, Inoue I, Ikeda M, et al. Statins activate human PPARalpha promoter and increase PPARalpha mRNA expression and activation in HepG2 cells. PPAR Res. 2008;2008:316306. doi:10.1155/2008/316306

57. Xu Y, Yang X, Wang Z, et al. Estrogen sulfotransferase (SULT1E1) regulates inflammatory response and lipid metabolism of human endothelial cells via PPAR $\gamma$. Mol Cell Endocrinol. 2013;369(1-2):140-149. doi:10.1016/j.mce.2013.01.020

58. Fruchart JC. Peroxisome proliferator-activated receptor-alpha (PPARalpha): at the crossroads of obesity, diabetes and cardiovascular disease. Atherosclerosis. 2009;205(1):1-8. doi:10.1016/j.atherosclerosis.2009.03.008

59. Yano M, Matsumura T, Senokuchi T, et al. Statins activate peroxisome proliferator-activated receptor gamma through extracellular signal-regulated kinase 1/2 and p38 mitogen-activated protein kinase-dependent cyclooxygenase-2 expression in macrophages. Circ Res. 2007;100(10):1442-1451. doi:10.1161/01.RES.0000268411.49545.9c

60. Sun L, Hu C, Zheng C, et al. Gene-gene interaction between CETP and APOE polymorphisms confers higher risk for hypertriglyceridemia in oldest-old Chinese women. Exp Gerontol. 2014;55:129-133. doi:10.2147/PGPM.S278671

61. Spirin V, Schmidt S, Pertsemlidis A, Cooper RS, Cohen JC, Sunyaev SR. Common single-nucleotide polymorphisms act in concert to affect plasma levels of high-density lipoprotein cholesterol. Am J Hum Genet. 2007;81(6):1298-1303.

62. Dagli-Hernandez C, Zhou Y, Lauschke VM, et al. Pharmacogenomics of statins: lipid response and other outcomes in Brazilian cohorts. Pharmacol Rep. 2021;74:47-66. doi:10.1007/s43440-021-00319-y

63. de Oliveira FF, Berretta JM, de Almeida Junior GV, et al. Pharmacogenetic analyses of variations of measures of cardiovascular risk in Alzheimer's dementia. Indian J Med Res. 2019;150(3):261-271. doi:10.4103/ijmr.IJMR_1209_17

Pharmacogenomics and Personalized Medicine

\section{Publish your work in this journal}

Pharmacogenomics and Personalized Medicine is an international, peer-reviewed, open access journal characterizing the influence of genotype on pharmacology leading to the development of personalized treatment programs and individualized drug selection for improved safety, efficacy and sustainability. This journal is indexed on the American Chemical Society's Chemical Abstracts Service (CAS). The manuscript management system is completely online and includes a very quick and fair peer-review system, which is all easy to use. Visit http://www. dovepress.com/testimonials.php to read real quotes from published authors.

Submit your manuscript here: https://www.dovepress.com/pharmacogenomics-and-personalized-medicine-journal 